\title{
DIALOGUE SYSTEM FOR ELDERLY PEOPLE CONSIDERING IMPRESSION FORMATION AND USER'S CHARACTER
}

\author{
Hiroshi Yajima, Huta Takeda, Jyunpei Kawaguchi and Manabu Kurosawa \\ Tokyo Denki University, 5 Asahi-cho Adachi-ku Tokyo Japan
}

\begin{abstract}
In recent years, lacks of conversation among elderly people are becoming a serious problem accompanying an increase in elderly living alone. For this trend, dialogue systems for solving the lack of conversation by elderly people have received attention. As elderly people are thought to like to talk, current dialogue systems for elderly people are specialized in listening to the story. However, it will be difficult for elderly people to speak positively to the dialogue systems from the beginning. In this paper, we propose a dialogue system that considers psychological factors such as communication process and user's character. In the proposed method, communication between elderly people and the dialogue system is divided into two stages. In the initial dialogue, an agent talks positively to make elderly people easy to talk. In the late dialogue, we propose two kinds of agents that adopt different dialogue methods according to the character of the user. Finally, we conducted experiments on the formation of impressions in the initial dialogue and verified the effectiveness of the proposed method.
\end{abstract}

\section{KEYWORDS}

Dialogue System, Elderly, Character, Impression Formation, Primacy Effect

\section{INTRODUCTION}

In recent years, Japan is entering an aging society. The proportion of elderly people aged 65 or over in Japan's total population is $27.3 \%$ (Cabinet Office, 2017). As the aging progresses, the proportion of elderly people is expected to become $33.3 \%$ in 2025 . In other words, it is said that one out of every three people will become an elderly person. With the progress of the aging population, the proportion of lonely elderly people is increasing. The proportion of elderly living alone in the elderly population was $4.3 \%$ for males and $11.2 \%$ for females in 1980. Thereafter, in men in 2001 the number increased to $13.3 \%$ for men and $21.1 \%$ for women. Senior citizens tend to be lacking in communication. We conducted a survey on the frequency of conversation among elderly people aged 60 and older. We analyzed those who were elderly people living alone and who responded that the frequency of conversation (including telephone and e-mail) was less than once every two or three days. As a result, the corresponding elderly people were $28.8 \%$ for males and $22.0 \%$ for females (Cabinet Office, 2016).

It is said that the risk of dementia is high in elderly people with poor communication. According to Saito et al. 'S research, the risk of dementia for elderly people whose frequency of conversation is less than once a week is 1.4 times that of elderly people who often talk (Dementia Forum, 2018).

In recent years, many chat dialogue systems for the elderly have been studied to solve the lack of communication of the elderly with aging (Aoyanagi et al., 2018), but the conventional system has the following problems: (1) system does not take into consideration factors such as the process of communication of the elderly and the character of the elderly, and (2) Because the system is not used to the elderly, it is difficult for the elderly to talk with the system that they first met.

The aim of this research is as follows. The first aim is to propose a system that is easy for the elderly to use, which has no dialogue experience with the dialogue system. The next aim is to propose a system that conducts dialogue in consideration of elderly personality. The effect of the proposed system is as follows: (1) the system eliminates the lack of communication of the elderly, and (2) the elderly has a good impression on the characters in the proposed system through conversation. Due to the effect of (2) above, higher elderly people can obtain higher degree of dialog satisfaction 


\section{RELATED RESEARCH}

Dialogue system research can be divided into two types. (1) a task - oriented dialogue system that achieves a goal, (2) a non - task - oriented dialogue system aimed at talking itself (Saito et al., 2015).

In recent years, a dialogue system research to solve the lack of communication among elderly people was proposed (Takuwa et al., 2017, Shin Nakakarumai et al., 2014). This system aims at conversation itself such as chatting and counseling for the elderly. Therefore, this system is classified as a non-task-oriented dialog system (Inaba et al., 2014).

The chat dialogue system for the elderly generally considers the characteristics of elderly people (Torishima et al., 2017, Kamiyama, 2018). Many of these systems are studied based on the reminiscence method which is psychotherapy (Yokoyama et al., 2006). The reminiscence is a psychotherapy advocated for American psychiatrist Robert Butler in the 1960s. An important element of the reminiscence method is that elderly persons will share memories of the past and others through speaking; the mental state of the elderly becomes stable.

It is well known that the retrospective method is effective for communication between the elderly and the system (Kamiyama 2018). For this reason, many chatting systems for the elderly listen to the remarks of the elderly, and adopt a system of striking the remarks of the elderly (in this paper, the interactive system is referred to as a listening interactive system) (Kurati et al., 2018,). In the listening interactive system, the response of the system consists of questions about elderly people's remarks or striking to the elderly's remarks. In other words, in the listening interactive system, the conversation between the elderly and the system progresses mainly by the elderly (Shitaoka et al., 2017).

However, elderly people do not always have a personality talking to the system. In order for the elderly to relax and talk themselves, it is also advisable for the system to provide an interesting topic. However, there are few studies on systems that voluntarily provide topics to elderly people. Also, there are many studies focusing on some characteristics of conversation. However, there is no research on the effect of the whole conversation.

\section{ISSUES OF LISTENING INTERACTIVE SYSTEM FOR THE ELDERLY}

As mentioned in Chapter 2, many researches on interactive dialogue for elderly people are research of chat dialogue system. The conventional interactive dialogue systems have the following three problems.

Issues (1): The process of communication is not considered in designing interactive dialogue system. In the case of dialogue between humans, there is a clear difference between the dialogue with the partner for the first time and the dialogue with a close friend. Also in the design of dialogue between the discourse system and the elderly, consideration of the communication process is important. Also, it is important for the discourse system to use the optimal dialogue method in each process to realize natural communication. However, in the conventional research, communication process is hardly considered in construction of discourse system.

Issues (2): It is difficult for the elderly to talk to the discourse system for the first time. In the case of an interactive dialogue system, the response of the system is composed primarily of incompatibility and questions. Therefore, it is necessary for the elderly to speak to the discourse system by themselves, but most elderly people do not have experience to dialogue with the discourse system. For this reason, when the elderly speaks for the first time with the discourse system, there may be a case of embarrassment on the part of the elderly, or elderly can not recognize the discourse system as a partner according to the character of the elderly. It is difficult for the elderly to speak to the discourse system autonomously when the initial dialogue between the elderly and the discourse system has begun.

Issues (3): There are elderly persons with a personality whose discourse system is difficult to talk to. For elderly people who prefer to talk about themselves, interactive methods of listening interactive systems are effective. However, there are some elderly people who do not like to talk about themselves. The conventional interactive dialogue system without considering the character of such elderly persons, is doubtful in effectiveness for all elderly people if the system uniformly respond to elderly. Also, it is sometimes necessary to change the conversation scenario of the dialogue system according to the mood of the elderly on the day. 


\section{PROPOSED METHOD}

\subsection{Purpose of Research}

In this paper, we propose a dialogue system incorporating a psychological point of view on the analysis of the communication process and the character of the elderly in order to solve the problem of the current state-of-the-art dialogue system described in Chapter 3.

\subsection{Outline of the Proposed System}

In this section, we present a proposed system to solve the three problems mentioned in Chapter 3 (Figure 1).

In order to solve the issue (1), the proposed system divides the communication process between the elderly and the discourse system into two stages. Specifically, the interaction process between the elderly and the discourse system is divided into two stages, "Initial Dialogue" and "Middle and Late Dialogue". And we developed a dialogue method suitable for each of these two stages.

In order to solve the issue (2), we developed an "initial dialog agent" to be used in the early stage of dialogue between the elderly and discourse system. Initial dialog agent adopts a dialogue form easy for the elderly to use a discourse system for the first time. This interactive format is constructed based on psychological knowledge. The aim of the initial dialog agent is to form a favorable impression on the discourse system for the elderly.

In order to solve the issue (3), we developed agents that consider the character of the elderly for discourse at the middle and late stages. Specifically, we developed two types of agents. These are "speech agents" who spontaneously talk to elderly, and the "listening agent" who listens frequently to elderly people. In the dis course of the middle and late stages, these two agents are selected according to the character of the elderly. In the proposed system, by switching such a discourse scheme, we provide a dialogue of a form that fits the elderly personality.

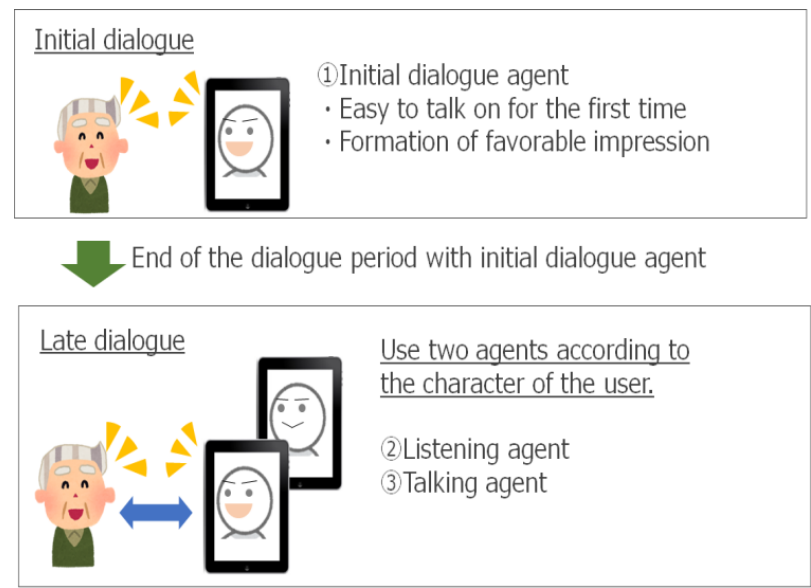

Figure 1. Image of the Proposed System

\subsection{Basic Policy of Initial Dialog Agent Development}

The purpose of the initial dialog agent is to provide an easy-to-talk environment for elderly people who are not accustomed to dialogue with the discourse system. Therefore, in the early stage discourse, basically, agent-based episode talk (hereinafter referred to as scenario response) is the main subject.

In the scenario response, the proposed system basically advances the dialogue in accordance with the scenario prepared in advance, so that the elderly will talk to the topic provided by the discourse system and answer the questions. In this way, dialogue will proceed. Through this initial discourse, even elderly people who are not used to dialogue with discourse system can easily adapt to dialogue with discourse system.

As an agent in the discourse system, we adopted "children in elementary school" because elderly people are naturally familiar with children. The scenario response has the following structure. First of all, the topic 
in discourse was an event in school. For elderly, the timings of dialogue with agent are twice in the morning and night in the day. In the morning dialogue, the agent makes simple greetings. In the evening dialogue the agent speaks to the elderly episodes about events at school.

\subsection{Change Method of Agent Scenario Response Using Elderly Input}

Basically, the scenario response of the initial dialog agent does not depend on the input contents of the elderly. However, if the input content of the elderly is different from one that is assumed in advance, the discourse system changes the response content of the agent according to the input contents of the elderly.

In this case, the agent's response corresponding to the case where the supposed input content matches the input contents of the actual elderly person is set in advance. With this setting, the agent can respond to the input contents of the elderly. In the case where the input content of the elderly does not exactly correspond to the input content assumed in advance, the discourse system classifies the input intention of the elderly into "other responses". Then, in that case, a reply with no sense of incompatibility is made regardless of the input contents of the elderly. For example, in the case where breakfast is the topic, agent replies "The doctor said that it is important to eat breakfast".

In addition, the discourse system not only uses the input contents of the elderly for the immediately following reply as described above, but also applies to the dialogue of the following day.

For example, if the agent makes a question saying "Have you eaten breakfast yet?", and the elderly answer is judged to be "not eating", the discourse system will record the responses of the elderly. Then, in the next morning, agent ask elderly "You seem to have not eaten breakfast yesterday, but did you eat today?"

By making such a response, the agent gives the elderly people the impression that the agent understands and remembers the elderly's talk answer.

\subsection{Gentleness Expression Function}

In order to realize improvement the satisfaction degree of dialogue which is one of the object of this research, ingenuity to improve the impression that the elderly holds against the agent is indispensable. Therefore, the initial dialog agent has a function to express gentleness, which is based on psychological knowledge.

Studies on interpersonal perception have revealed that there is a phenomenon called initial effect. Due to this phenomenon, the information presented at the initial stage of impression formation is found to have greater significance for the formation of impressions than the information given at a later time.

Various characteristics exist as personality characteristics of a human being as an element of impression formation. Among them, it has been clarified that characteristics having a central function exist in forming impressions such as "warmth", "cold", etc.

To summarize such findings, it can be said that a favorable impression is easily formed in the subsequent impression formation by giving the impression "gentle" at the beginning of impression formation.

Based on such knowledge, in order to improve the impression that the elderly has against the agent, we made the initial dialogue agent handling the initial dialogue function to express " gentleness ". " Gentleness " in this paper is defined as "compassion for others." Example of gentleness expression is to apply care-of words to others who are in trouble or sadness or make actions of gentleness.

As the method of initial dialog agent, there are two ways of expressing the gentleness expressed by the agent: "(1) expression of gentleness to the elderly" and "(2) expression of gentleness to other than the elderly." In (1) case, the agent responds such as worrying words when the elderly are in bad condition. In (2) case, the agent expresses gentleness by telling the elderly about the behavior to person other than the elderly such as "I helped a friend".

\section{AGENTS CONSIDERING THE CHARACTER OF THE ELDERLY}

\subsection{The Purpose of the Discourse Agent in the Latter Period of Discourse}

As shown in Section 4.2, the proposed system uses different dialogue methods in the early dialogue and the mid-latter term dialogue. In the middle-and-later term dialogue, the discourse system changes the dialogue method according to the character of the elderly. 


\subsection{The Purpose of the Discourse Agent in the Latter Period of Discourse}

There are various methods to classify the personality of elderly people, but in this paper "aggressiveness to dialogue" is used for character classification of the elderly. The grounds for this selection is made by the following two considerations.

(1) Other Personality Characteristics Classification affects emotional expression and change of ending in agent response.

(2) On the other hand, in the case of "aggressiveness to dialogue", the response of the agent affects the interactive form and the amount of dialogue. Therefore, influence on dialogue is strong using this method.

Specifically, using the utterance tendency scale which is a psychological measure, we classify the character of the elderly into "speaking liking" and "discreetness".

When using the proposed system, questionnaires are given to elderly subjects before system use, and elderly people are classified.

\subsection{Agent Classification}

We classified the elderly into two in terms of aggressiveness towards dialogue. In other words, we developed two types of agents in consideration of compatibility with individual elderly people: concretely, "listening agent" who makes dialogue with elderly people who are "talkative", and "Talking agent" that makes dialogue with "conservative" elderly people.

\subsection{The Purpose of the Discourse Agent in the Latter Period of Discourse}

The listening agent takes an interactive form suitable for the elderly with talkative character. Specifically, the listening agent performs utterance mainly about repetition and striking a match.

The flow of listening agent dialogue is shown below. 1) The elderly talks to the agent first. 2) The agent responds. 3) After the agent 's response, there are sometimes times when elderly people cannot find the topic of an appropriate conversation. At that time, when a certain period of time elapses, the agent automatically issues new questions related to the topic of the past to the elderly. 4) If there is a remark from the elderly and the previous system response is a new question, agent asks deep question such as "Why is that?" 5) Next, when the content of the elderly's speech is a question, the agent responds in an ambiguous manner to the question, and then a new question is subsequently made by agent. The reason why the agent responds ambiguously is that many questions from the elderly tend to be irrelevant to the current topic. As an agent, it is difficult to respond to everything to the remarks of elderly people irrelevant to topics.

Next, when the elderly's input matches a response rule including a specific word such as "I like AA", agent performs a parrots such as "You like AA". In addition, if the input of the elderly does not include a noun, etc., the agent makes a new question related to the topic in order to broaden the talk.

\subsection{Talking Agent}

The talking agent takes an interactive form suitable for elderly persons of modest character. Therefore, responses of agents are basically centered on scenario-like responses that do not depend on the input contents of elderly people.

As a dialog flow, firstly, the talking agent has performs processing of ambiguous response to the timer processing and makes the question like the listening agent. As a function other than that, the talking agent makes an utterance concerning the topic according to the scenario.

The scenario has a branch point. If the elderly 's response to the agent' s question matches the rule prepared in advance at that branch point, the agent responds to express empathy, self - disclosure, etc., and changes the topic. 


\section{CONFIGURATION OF THE PROPOSED SYSTEM}

This chapter describes the proposed system configuration (Figure 2). The proposed system uses MMD Agent from Nagoya Institute of Technology MMD Agent is a tool kit for spoke $n$ dialogue system integrating speech recognition, speech synthesis, 3D model display.

The feature of MMD Agent is that description of response rules in discourse and change of model can be easily performed. The proposed system uses MMD Agent as an interface. And we developed our own dialogue control system .using complicated response rules in cooperation with MMD Agent.

The dialogue control system consists of "initial dialogue agent", "talking agent", and "listening agent".
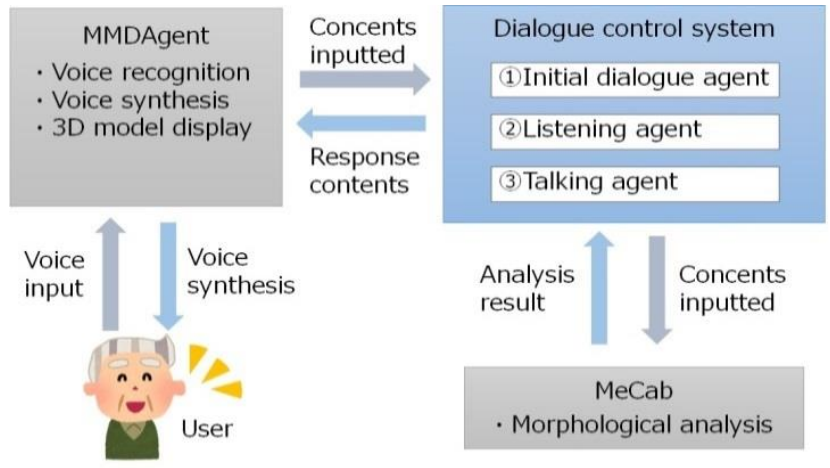

Figure 2. Configuration of the Proposed System

\section{EXPERIMENT}

\subsection{Purpose of Experiment}

The dialogue system proposed in this paper covers two kinds of dialogue between the elderly and discourse system (agent), that is, the initial dialogue and the middle and latter term dialogue. In this proposal, different dialogue methods are used for two kinds of dialogue. In this paper, we conducted experiments to verify the effectiveness of the initial dialogue agent used for the initial dialogue among the two types of dialogue. In the experiment, in particular, we verified that the "tenderness expression function" of the initial dialog agent influences the impression formation for the agent from the viewpoint of the elderly.

\subsection{Comparison System}

The purpose of the experiment is to verify the effectiveness of the gentleness expression function possessed by the initial dialog agent. For this reason, we used a typical conventional dialogue agent system (Kurati et al., 2018) as a comparison system.

\subsection{Experimental Procedure}

Eight people in their twenties who never used a dialogue system were taken as subjects. Subjects were divided into groups A and B. Group A used the proposed system and group B used the comparative system. The experimental period is 5 days and we conduct daily dialogue between morning and night. The average number of utterances in one dialog is 5 and the average dialog time is 1 minute 55 seconds. And we add three people in their eighties for our reference.

We conducted a questionnaire (Daibo edit, 1989, Hori edited, 2001) on the impression of the dialogue system for the subjects after the end of the dialogue on the first day night and the fifth day night of the experiment. The effectiveness of the proposed system is verified by comparing questionnaire results for each group and correlation analysis. 
Table 1. Analysis Result of the Questionnaire correlation analysis

\begin{tabular}{|l|c|c|c|}
\hline \multirow{2}{*}{ Question items } & \multicolumn{2}{|c|}{$\begin{array}{c}\text { Median of } \\
\text { evaluation }\end{array}$} & \multirow{2}{*}{$\begin{array}{c}\text { Correlation } \\
\text { coefficient }\end{array}$} \\
\cline { 2 - 4 } & A & B & \\
\hline 1: The agent is gentle & 5 & 3 & \\
\hline 2: Conversation is fun & 4.5 & 4 & 0.548 \\
\hline 3: Conversation can be relaxed & 4 & 3.5 & 0.287 \\
4: The agent is friendly & 5 & 4 & 0.523 \\
5: The agent understands a dialogue & 3 & 3 & 0.333 \\
\hline 6: Interaction is easyto talk & 3.5 & 3 & 0.738 \\
7: I'd like to talk to the agent more & 4 & 4.5 & 0.076 \\
8: The agent can be trusted & 3.5 & 3 & 0.491 \\
9: I am attached to the agent & 4.5 & 4.5 & 0.746 \\
\hline
\end{tabular}

\subsection{Experimental Result}

The results of the questionnaire were tabulated for each group (Table 1). Questionnaires were also analyzed by the following procedure.

(1) Impression analysis on the tenderness of agent response. First, by using the gentleness expression function, it is necessary to verify whether the subject evaluated the character of the agent as gentle. We compared the effectiveness of gentleness expressions in groups A and B by "1" item of .table 1 (Subject thinks the agent is a gentle personality). As a result, the evaluation value of group A using the proposed system was median value 5, and the evaluation value of group B using comparison system was median value 3. From this result, by using the gentleness expression function, we found that subject could feel agent more gentle.

(2) Correlation analysis of gentleness and other impression formation rank correlation analysis was carried out to verify the correlation between the impression of the agent and the impression of other factors. The definition and calculation method of the two variables used in the analysis are shown below.

[Variable 1]: Evaluation of the gentleness that subject felt during the dialogue period [Calculation method]: With respect to the evaluation of question 1, the average value (value by subject) on the first day and the fifth day.

[Variable 2]: Improvement degree of each impression evaluation during the experiment period [Calculation method]: For questions other than question 1, the difference between day 1 and day 5 (value by subject).

As a result of the questionnaire, it was found that questions 6 and 9 had a strong correlation, and questions 2, 4 and 8 had a strong correlation.

\section{DISCUSSION}

As a result of the impression evaluation questionnaire, the proposed system was gained high overall evaluation. From the interview after the questionnaire, it became clear that the subject recognized the agent as a gentle character in the initial dialogue. Based on this recognition, it is considered that favorable impressions were formed as a whole discourse in subjects.

Even in the experiment, as a result of the correlation analysis, it was found that giving the impression that the agent is a gentle character at the beginning of the dialogue has a strong correlation with ease of talking and attachment formation.

The index of ease of talking is a very important index for users without dialogue experience with the dialogue system. In addition, the index of whether or not you can afford attachment is also very important in considering long-term communication with the dialogue system. So, It is considered that the usefulness of the "gentleness expression function" proposed in this paper is high. As the evaluation of items 6, 8, 9 was high, it became clear that issue 1 was solved. From items 1 and 4, it became clear that the division of the communication process was effective. From items 2, 3, 5, and 7 it became clear that issue 3 was solved. 


\section{CONCLUSION}

In this paper, we proposed a discourse system for conversation closely to the elderly in order to solve the problem of the current dialogue system for elderly people. Specifically, we proposed a system to promote dialogue with emphasis on the feelings of elderly people. Specifically, the communication was divided into two. The initial phase is the initial dialogue phase impressing the tenderness of the agent.

From the experimental results, it became clear that 1) subjects received an expression of the gentleness of the agent, and a familiar relationship was born between the subject and the agent in the initial conversation, 2) the continuation of the conversation became smooth.

Future tasks are as follows.

1) Confirming the effectiveness when subjects are elderly

2) To clarify the individual effects with regard to the introduced multiple functions

3) Realize communication using scenarios considering emotion of elderly people.

\section{REFERENCES}

Aoyanagi, S. et al., 2018, Analysis of user's utterance to reduce inappropriate response of chat robot, proceeding of JSAI2018, pp. 234-238

Breuer, H. et al., 2008, A Mobile Pattern Discourse System, IEICE MoMuC 107(446), pp. 7-12

Cabinet Office, 2016, Aged Society White Paper http://www8.cao.go.jp/kourei/whitepaper/ w-2015/html/zenbun/index.html (Last accessed date: Feb. 11 2019)

Cabinet Office, 2017, elderly society white paper, http://www8.cao.go.jp/kourei/whitepaper/ w-2017/html/zenbun/index.html (Last accessed date: Feb. 11 2019)

Daibo, I. edit, 1989: "Social Psychology Perspective 1", SeiShin Shobo, Tokyo.

Dementia Forum, 2018, https://www.ninchisho-forum.com/knowledge/iryou/008.html (Last accessed date: Feb. 11 2019)

Hori, Y. edited, 2001, "Psychometric Measure Scale I", Science,Tokyo.

Inaba, M. et al., 2014, Candidate Utterance Acquisition Method for Non-task-oriented Dialogue Systems from Twitter, Journal of Artificial Intelligence Society, Vol.29, No.1, pp. 21-31

Kamiyama, T. 2018, Conversation service so much by heart, practical report of Information processing society of japan, 9(2), pp. 437-453.

Kurati, Y. et al., 2018, Chatbot Conversation Techniques, Fujitsu Journal 69(4), pp. 57-62.

Saito, M. et al., 2015, Criteria for social isolation based on associations with health indicators among older people. A 10-year follow-up of the Aichi Gerontological Evaluation Study, Japan Public Health Magazine, Vol.62, No.3, pp. $95-105$

Shin, N. et al., 2014, Development of voice conversation system to automate response for elderly living alone: Prediction of response timing using multiple regression analysis based on prosodic information and power, utterance interval, IEICE technical report, 113(481), pp. 19-23

Shitaoka, K. et al., 2017, Active listening system for a dialogue robot”, Natural language processing, Vol. 24, No.1, pp. 3-47

Takuwa, H. et al., 2017, A Study on Utterance Method Considering User's Hobby in Chat Dialogue System, proceeding of JSAI2017, pp. 212-217,

Torishima, M. et al., 2017, A Study on Chat Bot-based Communication, IEEJ Technical Report, Tottori Japan pp. 7- 9

Yokoyama, T. et al., 2006, Lessons learned from student 's visit activities for welfare, the Japanese association of rural medicine, 55(0), pp. 129-130. 